MINI-SYMPOSIUM

\title{
Pericardial constriction: uncommon patterns
}

\author{
J Sagristà-Sauleda
}

Heart 2004;90:257-258. doi: 10.1136/hrt.2003.024828

C onstrictive pericarditis can be defined as a syndrome (or syndromes) resulting from compression of the heart caused by rigid, thickened, and frequently fused pericardial membranes. This syndrome was known about more than three centuries ago, but more recently its clinical spectrum has changed in two ways. Firstly, from the aetiologic point of view, there has been an increase in the number of cases of constrictive pericarditis secondary to chest radiation and cardiac surgery. In particular, cardiac surgery has emerged as an important cause of constrictive pericarditis, representing up to $18-29 \%$ of cases in some series, ${ }^{12}$ although its prevalence is only $0.2-0.3 \%$ following coronary artery bypass grafting or valvar surgery. Secondly, the haemodynamic spectrum has been expanded with other forms of constriction. In this paper we will first comment on the classical syndrome of chronic constrictive pericarditis, and then describe the other less known syndromes of cardiac constriction. Finally, some aetiological considerations will be discussed.

\section{CHRONIC CONSTRICTIVE PERICARDITIS}

Chronic constrictive pericarditis is caused by a rigid, shell-like pericardial scar that restricts ventricular filling to earlier diastole. The clinical picture includes chronic fatigue and dyspnoea, neck vein distension with a brisk diastolic collapse (" $\left.\mathrm{y}^{\prime \prime}\right)$ of the jugular venous pulse, pericardial knock, enlarged liver, ascites, peripheral oedema, and pleural effusion. Arterial pulsus paradoxus is usually absent. Atrial fibrillation is present in half of the patients. Constrictive pericarditis should be suspected in all patients with findings suggestive of right heart failure or ascites; diagnosis is usually not difficult provided there is an adequate index of suspicion. Diagnosis should be based on a triad of: a suggestive clinical syndrome; demonstration of a physiology of constriction/ restriction; and demonstration of a thickened pericardium. Demonstration of constriction/restriction is usually accomplished by Doppler echocardiography, which can show characteristic findings. ${ }^{3}$ A thickened pericardium can be apparent in a simple chest $x$ ray in cases with pericardial calcification (present in about $30-50 \%$ of the cases), or in computed tomography or magnetic resonance imaging. It should be emphasised that Doppler echocardiographic signs are not $100 \%$ accurate, and that the pericardium may seem apparently normal using other imaging techniques, but this occurrence is very rare in chronic constrictive pericarditis. In fact, the need to perform an exploratory thoracotomy to make a differential diagnosis of restrictive cardiomyopathy rarely arises. Although different aetiologic types of pericarditis may eventually end in a constrictive pericarditis, histologic study of excised pericardium shows non-specific findings in the vast majority of cases of chronic constrictive pericarditis.

\section{UNCOMMON PATTERNS OF CARDIAC CONSTRICTION}

For centuries, chronic constrictive pericarditis was the only known syndrome of cardiac constriction. More recently, other syndromes of cardiac constriction have been described, which share the characteristics of being less severe or having a shorter clinical course. These types of constrictive pericarditis frequently exhibit a haemodynamic pattern of "elastic"4 rather than rigid constriction, which in some way is a compressive syndrome intermediate between tamponade and rigid constriction.

\section{Effusive-constrictive pericarditis}

Effusive-constrictive pericarditis is a clinical-haemodynamic syndrome in which there is constriction of the heart by the visceral pericardium in the presence of effusion in free pericardial space. This type of constrictive pericarditis was first characterised from the haemodynamic point of view by Hancock $^{5}$ in his report on 13 patients submitted to pericardiectomy. The hallmark of effusive-constrictive pericarditis is the demonstration of persistently raised right atrial and end diastolic ventricular pressures after the intrapericardial pressure is reduced to normal levels by removal of pericardial fluid. In our experience over a period of 16 years, the prevalence of this disorder is only $1.3 \%$ among patients with pericardial disease and around $6.8 \%$ among patients who present with clinical tamponade. ${ }^{6}$ The aetiologic spectrum includes idiopathic cases, together with cases subsequent to chest radiation or cardiac surgery, or caused by neoplasia or tuberculosis. Patients with effusive-constrictive pericarditis usually show a subacute evolving clinical course, with inflammatory symptoms and signs often present. On admission clinical findings correspond to tamponade. The presence of additional constriction is frequently overshadowed, although some findings on Doppler echocardiography can alert the clinician to the condition. After resolution of tamponade by pericardiocentesis, these findings can become more apparent together with clinical findings of constriction (prominent " $\mathrm{y}$ " collapse, $\mathrm{S}_{3}$ sound). Probably most cases of effusive-constrictive pericarditis will eventually evolve to persistent constriction requiring pericardiectomy. At surgery attention must be paid to the visceral layer of the pericardium with an adequate attempt at epicardiectomy. However, effusive-constrictive pericarditis may be transient and may resolve spontaneously, especially in idiopathic cases.

\section{Transient cardiac constriction}

The possibility that a cardiac constriction could be a transient phenomenon was unrecognised until the description by our group in 1983 and later in $1987 .{ }^{7}$ We reported on 16 patients from a series of 177 with effusive acute idiopathic pericarditis in which features of constriction were detected in the phase of resolution of pericarditis, at a time when signs of activity had abated and effusion was already minimal or had disappeared entirely. Clinical (pericardial knock on auscultation, " $y$ " collapse in the jugular venous pulse) and echocardiographic (early diastolic septal notch) features of constriction were frequently present, and two patients had overt signs of venous congestion. In five patients, cardiac catheterisation was carried out and disclosed features of constrictive pericarditis in all, either in the baseline state or 
after fluid challenge. After a mean period of 2.7 months the clinical and haemodynamic features of constriction spontaneously subsided. The possibly transient character of cardiac constriction is not only of theoretical interest; awareness of it may obviate the need for pericardiectomy. In addition, the finding of transient constriction expands the range of possibilities from acute effusive pericarditis, and illustrates the fact that a continuum of different outcomes can be considered to exist. Although most patients with acute pericarditis proceed directly to complete resolution, in some recovery may be preceded by transient constriction; other patients may develop subacute effusive-constrictive pericarditis, sometimes followed by classical constrictive pericarditis, or their condition may directly progress to subacute or chronic constriction. Transient constriction would represent an intermediate link between uncomplicated recovery and the severe, irreversible types of constriction.

\section{Occult constrictive pericarditis}

The term occult constrictive pericarditis was introduced in 1977 by Bush and associates. ${ }^{8}$ They reported on 19 patients, most with idiopathic pericarditis, in whom physical and haemodynamic features of constriction were not apparent in their baseline state, but which were brought about by the rapid infusion of saline. However, we caution against routinely administering saline to all patients with imprecise symptoms being evaluated for pericardial disease, as fluid administration is a poorly standardised test and the interpretation of the findings can be quite difficult. At present, we rarely perform fluid challenge in everyday practice, and we have never indicated pericardiectomy solely on the basis of this procedure.

\section{PATTERNS OF CARDIAC CONSTRICTION IN DIFFERENT AETIOLOGIES OF PERICARDIAL DISEASE}

In a prospective follow up of patients with acute pericardial disease, ${ }^{9}$ severe subacute constriction requiring pericardiectomy developed in 56\% (9/16) and 35\% (6/17) of patients with tuberculous and purulent pericarditis, respectively, and in $17 \%(2 / 12)$ of patients with neoplastic pericarditis. In contrast, only 2 of 177 patients with acute idiopathic pericarditis developed effusive-constrictive pericarditis requiring pericardiectomy. In all of them, constriction was subacute, developing within the first six months of follow up.
Thus, a patient with subacute constriction after acute pericardial disease is much more likely to have a specific pericarditis than an idiopathic pericarditis. On the other hand, transient constriction is much more frequent in patients with idiopathic pericarditis (with a prevalence of $20 \%$ when specifically sought) than in tuberculous or purulent pericarditis. Thus, a gradient of severity of cardiac constriction can be assumed, from severe subacute forms to transient constriction. This gradient would be weighted to the severe side in cases of tuberculous, purulent or neoplastic pericarditis, where severe types of permanent constriction predominate, and to the mild side in idiopathic pericarditis, where benign transient constriction is the rule. By contrast, none of the 28 patients with massive chronic idiopathic pericardial effusion developed any feature of constriction. ${ }^{10}$ These patterns of constriction in the different aetiologies of pericardial disease may have some clinical value for the diagnosis in individual patients.

Correspondence to: Dr J Sagristà-Sauleda, Servei de Cardiologia, Hospital Universitari Vall d'Hebron, Pg. Vall d'Hebron 119-129, 08035 Barcelona, Spain; isagrist@vhebron.net

\section{REFERENCES}

1 Ling LH, Oh JK, Schaff HV, et al. Constrictive pericarditis in the modern era. Evolving clinical spectrum and impact on outcome after pericardiectomy. Circulation 1999:100:1380-6.

2 Cameron J, Oesterle SN, Baldwin JC, et al. The etiologic spectrum of constrictive pericarditis. Am Heart J 1987; 113:354-60.

3 Candell-Riera J, Garcia-del-Castillo H, Permanyer-Miralda G, et al. Echocardiographic features of the interventricular septum in chronic constrictive pericarditis. Circulation 1978;57:1 154-8.

4 Hancock EW. On the elastic and rigid forms of constrictive pericarditis. Am Heart J 1980;100:917-23.

5 Hancock EW. Subacute effusive-constrictive pericarditis. Circulation 1971;43:183-92.

6 Sagristà-Sauleda J, Angel J, Sänchez A, et al. Effusive-constrictive pericarditis. N Engl J Med 2004;350:469-75.

7 Sagristà-Sauleda J, Permanyer-Miralda G, Candell-Riera J, et al. Transient cardiac constriction: un unrecognized pattern of evolution in effusive acute idiopathic pericarditis. Am J Cardiol 1987;59:961-6.

8 Bush CA, Stang JM, Wooley CF, et al. Occult constrictive pericardial disease. Diagnosis by rapid volume expansion and correction by pericardiectomy. Circulation 1977;56:924-30.

9 Permanyer-Miralda G, Sagristà-Sauleda J, Soler-Soler J. Primary acute pericardial disease: a prospective series of 231 consecutive patients. Am J Cardiol 1985;56:623-30.

10 Sagristà-Sauleda J, Angel J, Permanyer-Miralda G, et al. Long-term follow-up of idiopathic chronic pericardial effusion. N Engl J Med 1999;341:2054-9.

\section{ELECTRONIC PAGES}

\section{Heart Online case reports: www.heartjinl.com}

$\square$ he follow electronic only articles are published in conjunction with this issue of Heart.

\section{Massive pulmonary haemorrhage complicating the treatment of acute coronary syndrome}

\section{S Gill, K Ng, K-S Ng}

Antiplatelet medications such as clopidogrel and tirofiban (glycoprotien IIb/IIIa inhibitors) are associated with a reduction in mortality and morbidity and are therefore recommended in the treatment of acute coronary syndromes. Massive pulmonary haemorrhage is a rare complication of both clopidogrel and tirofiban. Pulmonary haemorrhage may easily be mistaken for acute pulmonary oedema, a condition commonly seen in patients with acute coronary syndrome. Physicians need to be aware of this diagnostic dilemma because early treatment increases the chances of patient survival.

(Heart 2004;90:e15) www.heartjnl.com/cgi/content/full/90/ 3/e15

\section{Haemorrhagic peritonitis as a late complication of echocardiography guided pericardiocentesis H Luckraz, S Kitchlu, A Youhana}

Clinically significant pericardial effusion is an uncommon complication after cardiac surgery. Pericardiocentesis can be performed either through a mini-sternotomy or under echocardiography guidance. Echocardiography guidance is a relatively safe procedure and it avoids the need for another general anaesthetic. However, in this post cardiac surgical patient echocardiography guided pericardiocentesis was complicated several days later by haemorrhagic peritonitis.

(Heart 2004;90:el6) www.heartjnl.com/cgi/content/full/90/ 3/e16 